\title{
Epigenetic Therapeutic Strategies to Target Molecular and Cellular Heterogeneity in Pancreatic Cancer
}

\author{
Lennart Versemann $^{a} \quad$ Elisabeth Hessmann ${ }^{a, b} \quad$ Maria Ulisse $^{a, b}$ \\ aDepartment of Gastroenterology, Gastrointestinal Oncology and Endocrinology, University Medical Center

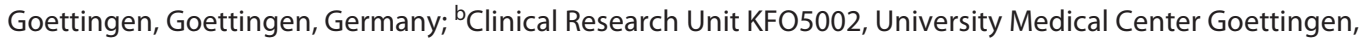 \\ Goettingen, Germany
}

\section{Keywords}

Epigenetics · Pancreatic cancer · Subtype · Tumor heterogeneity

\begin{abstract}
Background: Pancreatic ductal adenocarcinoma (PDAC) remains a major challenge in cancer medicine and is characterized by a 5 -year survival rate of $<10 \%$. Compelling evidence suggests that the devastating disease outcome of PDAC patients is linked to a high degree of intra- and interindividual tumor heterogeneity, which is predominantly installed at the level of gene transcription. The cellular and molecular complexities of the disease explain the poor efficacy of "onesize-fits-all" therapeutic approaches in PDAC treatment and strongly argue for pursuing tailored therapeutic strategies to tackle PDAC. In a highly dynamic manner, a network of transcription factors and epigenetic regulatory proteins temporally and spatially control the diverse transcriptomic states determining PDAC heterogeneity. Given the reversibility of epigenetic processes, pharmacological intervention with key epigenetic drivers of PDAC heterogeneity appeals as a promising concept to shift the transcriptomic phenotype of PDAC toward a less aggressive and more chemosensible state. Summary: In this review, we discuss the chances and pitfalls of epigenetic treatment strategies in overcoming and shifting molecular and cellular PDAC heterogeneities in order to combat PDAC. To this end, we utilized the keywords "pancreatic cancer," "heterogeneity," and "epigenetics" to search for relevant articles on the database PubMed and selected interventional studies enrolling PDAC
\end{abstract}

karger@karger.com www.karger.com/vis

Karger ${ }^{\prime \prime}=$ BOPEN ACCESS
(C) 2021 The Author(s)

Published by S. Karger AG, Basel

This article is licensed under the Creative Commons Attribution 4.0 International License (CC BY) (http://www.karger.com/Services/ OpenAccessLicense). Usage, derivative works and distribution are permitted provided that proper credit is given to the author and the original publisher. patients as displayed in clinicaltrails.gov to generate a synopsis of clinical trials involving epigenetic targeting. Key Messages: Targeting epigenetic regulators in PDAC represents a promising concept to reprogram molecular and cellular tumor heterogeneities in the pancreas and hence to modulate the PDAC phenotype in favor of a less aggressive and more therapy susceptible disease course. However, we just start to understand the complex interactions of epigenetic regulators in controlling PDAC plasticity, and a clinical breakthrough utilizing epigenetic targeting in PDAC patients has not been achieved yet. Nevertheless, increasing translational efforts which consider the pleiotropic effects of targeting epigenetic regulation in different cellular compartments of the tumor and that focus on the utility and sequence of combinatory treatment approaches might pave the way toward novel epigenetic treatment strategies in PDAC therapy.

(c) 2021 The Author(s).

Published by S. Karger AG, Basel

\section{Introduction}

Pancreatic ductal adenocarcinoma (PDAC) remains a major challenge in cancer medicine. Indeed, despite remarkable efforts in translational research and drug development, the overall 5 -year survival rate of $<10 \%$ of patients has remained unchanged for almost 20 years [1]. Major causes for the dismal outcome are the exceptionally aggressive tumor biology with early onset of metastasis and the remarkable resistance to conventional chemotherapy. Tumor cell progression and therapy evasion 
processes are driven by a high degree of cellular and molecular tumor heterogeneity [2]. Increasing evidence suggests that PDAC heterogeneity is determined by transcriptomic phenotypes, which are hierarchically installed and controlled by epigenetic cues [2-8]. Epigenetic regulatory proteins converge on the transcriptional landscape by controlling, for example, chromatin accessibility, DNA methylation, and histone modification, thus finetuning the transcriptional output of a given cell in a spatially and temporally restricted manner [9]. The dynamic nature and the reversibility of epigenetic processes characterize epigenetic regulatory proteins as promising targets to shift transcriptional phenotypes of cancer cells toward less aggressive and more therapy susceptible states. In this review, we discuss conceptual and translational efforts exploiting epigenetic targeting for PDAC treatment with a particular focus on the consequences of epigenetic reprogramming on the molecular and cellular heterogeneity of the disease.

\section{Targeting Epigenetics to Interfere with Molecular PDAC Heterogeneity}

\section{Molecular PDAC Subtypes}

Given the advances in next-generation sequencing technologies and motivated by the successes of molecular stratification-based treatment approaches in other tumor entities [10-12], the last decade has witnessed a plethora of whole-genome sequencing studies and transcriptional profiling analyses conducted in large cohorts of PDAC tumors aiding at the dissection of the molecular landscape of PDAC $[2-8,13]$. These studies did not only reaffirm signature mutations in KRAS, TP53, CDKN2A, and $S M A D 4$ but led to the identification of numerous additionally mutated or transcriptionally altered genes. Importantly, the molecular heterogeneity of PDAC is reflected in the identification of various molecular and phenotypic PDAC subtypes with prognostic and therapy predictive significance $[3-5,14]$. Despite discrepancies in the definition of molecular PDAC subtypes, transcriptome and epigenome analyses consistently identified 2 major lineages which separate PDAC into basal-like (also called "squamous" or "quasi-mesenchymal") and classical (also considered as "progenitor-like") subtypes [2-8, $13,14]$. While the basal-like subtype is associated with a high tumor grade, strong chemoresistance, and the worst prognosis $[3-5,14]$, classical subtype tumors are better differentiated, associated with improved responsiveness to chemotherapy and a better prognosis $[3,4,14]$. Molecularly, these subtypes are linked to distinct gene signatures and epigenetic profiles $[8,15]$. While the basal-like subtype shows a more mesenchymal expression profile, the classical subtype comprises an epithelial differentia- tion gene signature $[2-8,13,14]$. Moreover, the 2 subtypes differ in the activity of distinct superenhancers and their upstream regulators [8]. Superenhancers operate as regulatory elements known to have a huge ability to influence target gene expression, to have cell- and state-specific activities, and to be bound by lineage-defining transcription factors $[16,17]$. The most prominent transcription factors regulating subtype-specific superenhancers and transcription programs are GATA6, PDX1, and $\mathrm{HNFs}$ for the classical, and MET, MYC, and the $\triangle \mathrm{N}$ isoform of the transcription factor TP63 $(\Delta \mathrm{Np} 63)$, for the basal-like state $[8,15,18-20]$. Importantly, compelling evidence suggests that epigenetic regulators complement and control the activity of these subtype-determining transcription factors, either by influencing their expression or by acting as transcriptional co-regulators [20,21]. Given the dynamic character of these drivers of PDAC subtype identity, the distinct subtype states are not permanently installed but underlay a high degree of plasticity. Considering the better prognosis and the increased chemosensitivity of classical versus basal-like PDAC subtypes $[3-5,14]$, the concept of subtype switching seems to be a highly appealing strategy, for example, preceding cytotoxic PDAC therapy. Consistent with this idea, many translational approaches in PDAC aim at deciphering strategies to induce subtype switching. Given the reversibility of epigenetic regulations, pharmacological interference with epigenetic key regulators of PDAC subtype identity has moved into the focus of approaches aiding at molecular PDAC heterogeneity.

\section{Epigenetic Targeting Strategies to Induce Subtype \\ Switching in PDAC}

Above others, the endodermal lineage transcription factor GATA6 has been characterized as a hierarchical regulator of classical PDAC subtype identity $[4,8,22]$ and as a robust surrogate biomarker for differentiating classical (GATA6 ${ }^{\text {high }}$ ) and basal-like (GATA6 ${ }^{\text {low }}$ ) PDAC subtypes [14]. Consistently, depletion of GATA6 is necessary to induce the basal subtype-specific transcription factor $\triangle \mathrm{Np} 63$ and to enforce a basal-like PDAC subtype state [22]. Hence, pharmacological approaches inducing or stabilizing GATA6 expression qualify as promising strategies to push PDAC cells toward the classical PDAC subtype. The potential of GATA6 induction for enforcing classical PDAC subtype identity was introduced by a study of Lomberk et al. [8]. Herein, the authors demonstrate that inhibition of the basal-like subtype-specific superenhancer regulator MET induces a basal-like-to-classical subtype switch via transcriptional activation of GATA6 and subsequent induction of GATA6-dependent gene regulation [8]. Along the same line of evidence, loss of the basal-like subtype-specific transcription factor GLI2, which is involved in Hedgehog signaling [23], re- 


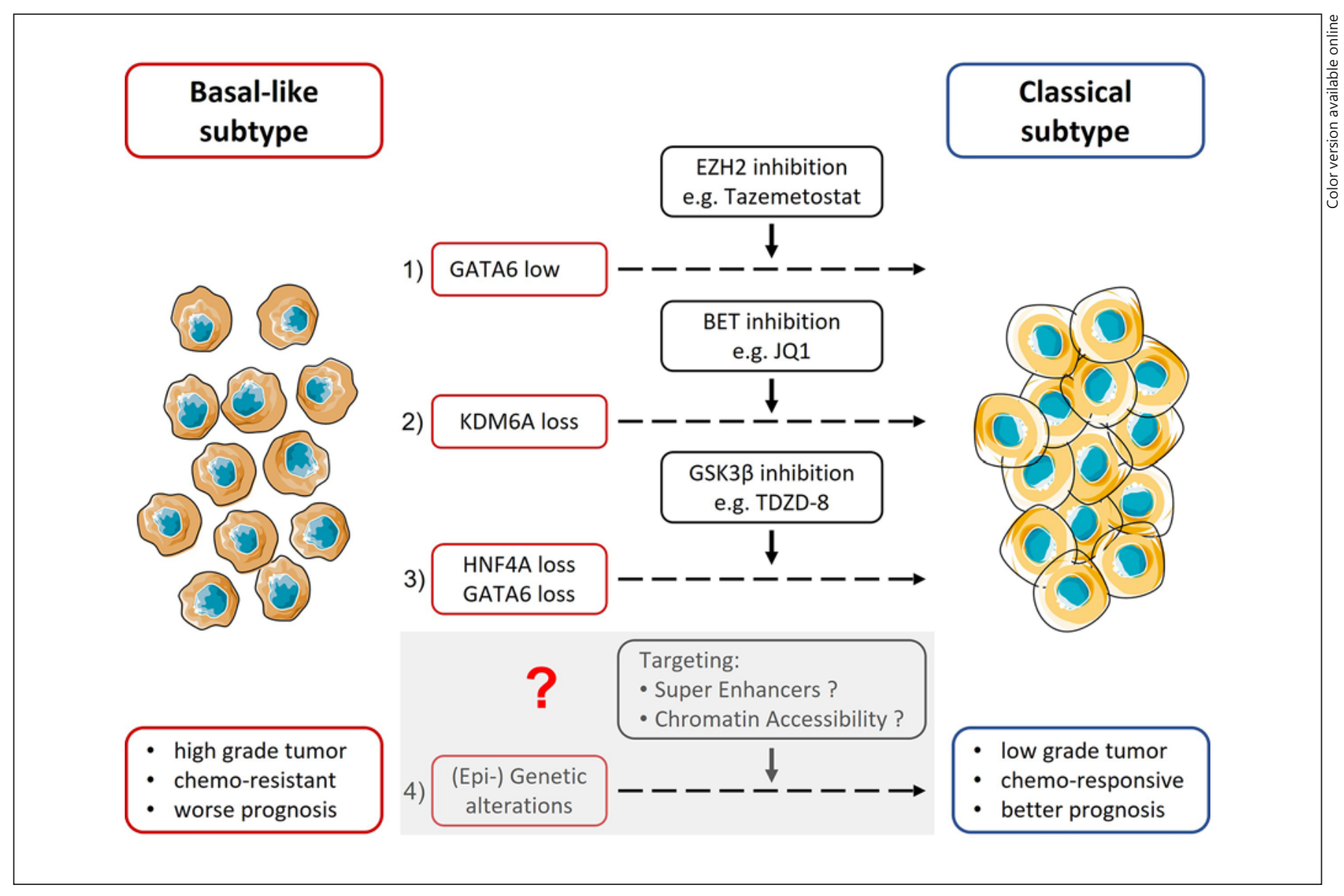

Fig. 1. Epigenetic targeting strategies to tackle PDAC subtype identity. PDAC can be classified into the classical and basal-like subtypes differing in chemosensitivity and prognosis. However, there is a dynamic plasticity between these 2 PDAC subtypes. Therefore, forcing a subtype switch from the aggressive basal-like subtype into the less aggressive classical subtype might be a compelling therapeutic option. (1) Derepression of GATA6 expression upon EZH2 inhibition (e.g., using tazemetostat) [21]; (2) BET inhibition (e.g., using JQ1) in KDM6A-deficient PDAC [20]; and (3)
GSK3 $\beta$ inhibition (e.g., using TDZD-8) PDAC subtypes characterized by low expression of GATA6/HNF4A [26]. (4) Prospectively, it can be hypothesized that targeting superenhancer activity or chromatin accessibility might be an appealing approach to target PDAC subtype identity upon molecular stratification of the tumor $[8,26,57]$. Designed with https://smart.servier.com/. PDAC, pancreatic ductal adenocarcinoma; BET, bromodomain and extra-terminal motif; DNMT, DNA methyltransferase. sults in increased GATA6 expression and acquisition of classical gene signatures in PDAC [24]. While these reports emphasize the role of subtype-specific transcription factors in regulating the expression of their classical counterpart GATA6, Patil et al. [21] recently identified the histone methyltransferase enhancer of zeste homolog 2 $(\mathrm{EZH} 2)$ as a direct transcriptional regulator of GATA6 in PDAC. In accordance with its activity as a transcriptional repressor, EZH2 binding to the GATA6 TSS region silenced GATA6 transcription, thus promoting PDAC invasion and metastasis. Interestingly, blockade of EZH2 activity was sufficient to reinstall GATA6 expression and to induce gene signatures associated with the classical PDAC subtype state. Hence, these data reveal pharmacological interference with EZH2-dependent GATA6 repression as a promising strategy to induce subtype switch- ing, restrain tumor progression, and increase chemosensitivity in PDAC (Fig. 1) [21]. However, given the existence of PDAC tumors with EZH2-independent GATA6 regulation [1], EZH2 targeting might only be beneficial in a subgroup of GATA6 ${ }^{\text {low }}$ PDAC subtypes, thus arguing for molecular stratification of GATA6 expression prior to application of EZH2 inhibitors. Nevertheless, the recent FDA approval of the EZH2 inhibitor tazemetostat for the treatment of advanced epithelioid sarcoma and a clinical trial exploring the drug in solid tumor entities including PDAC (NCT04705818, Table 1) suggest a potential clinical relevance of the identified EZH2-GATA6 axis in PDAC (Fig. 1).

In addition to a simultaneous loss of GATA6, epigenetic silencing of $H N F 4 A$ and $H N F 1 A$, has been lately reported as a prerequisite for basal-like subtype identity 
Table 1. Currently active clinical trials involving epigenetic treatment strategies in PDAC

\begin{tabular}{|c|c|c|c|c|c|c|}
\hline NCT number & Status & Drug & Co-treatment & Target & Phase & Enrolled tumor entities \\
\hline NCT03264404 & Recruiting & Azacitidine & Pembrolizumab & DNMT1 & 2 & Pancreatic cancer \\
\hline NCT01845805 & $\begin{array}{l}\text { Active, not } \\
\text { recruiting }\end{array}$ & Azacitidine & Abraxane, gemcitabine & DNMT1 & 2 & Pancreatic cancer \\
\hline NCT04257448 & Recruiting & $\begin{array}{l}\text { Azacitidine, } \\
\text { romidepsin }\end{array}$ & $\begin{array}{l}\text { Nab-paclitaxel + } \\
\text { gemcitabine, durvalumab + } \\
\text { lenalidomide capsule }\end{array}$ & DNMT1, HDAC class I & $1 / 2$ & Pancreatic cancer \\
\hline NCT03250273 & $\begin{array}{l}\text { Active, not } \\
\text { recruiting }\end{array}$ & Entinostat & Nivolumab & HDAC class I & 2 & $\begin{array}{l}\text { Pancreatic cancer, } \\
\text { cholangiocarcinoma }\end{array}$ \\
\hline NCT01638533 & $\begin{array}{l}\text { Active, not } \\
\text { recruiting }\end{array}$ & Romidepsin & None & HDAC class I & 1 & $\begin{array}{l}\text { Pancreatic cancer and other } \\
\text { solid tumor entities, } \\
\text { hematological malignancies }\end{array}$ \\
\hline NCT04705818 & $\begin{array}{l}\text { Not yet } \\
\text { recruiting }\end{array}$ & Tazemetostat & Durvalumab & $\mathrm{EZH} 2$ & 2 & $\begin{array}{l}\text { Pancreatic cancer and other } \\
\text { solid tumor entities }\end{array}$ \\
\hline NCT02349867 & $\begin{array}{l}\text { Active, not } \\
\text { recruiting }\end{array}$ & Vorinostat & $\begin{array}{l}\text { Gemcitabine + sorafenib + } \\
\text { chemoradiation }\end{array}$ & HDAC class I/II & 1 & Pancreatic cancer \\
\hline NCT03878524 & Recruiting & Vorinostat & $\begin{array}{l}52 \text { drugs (chemotherapy, } \\
\text { small inhibitors, antibodies) }\end{array}$ & HDAC class I/II & 1 & $\begin{array}{l}\text { Pancreatic cancer and other } \\
\text { solid tumor entities, } \\
\text { hematologic malignancies }\end{array}$ \\
\hline
\end{tabular}

PDAC, pancreatic ductal adenocarcinoma; DNMT, DNA methyltransferase; HDAC, histone deacetylase. Selection criteria applied in clinicaltrails.gov: interventional study type, pancreatic cancer, epigenetic treatment involved.

[22]. Loss of HNF4A favors upregulation of GSK3 $\beta$, which promotes metabolic programs in accordance with the basal-like PDAC subtype [25]. Interestingly, basallike PDAC subtypes were more sensitive to pharmacological inhibition of GSK3 $\beta$ (Fig. 1) [26]. However, the fact that a subgroup of PDAC developed resistance toward GSK3 $\beta$ inhibition [26] suggests the existence of subgroups within the basal-like PDAC subtype state, whose therapeutic utilities remain to be further explored.

Subtype switching induced by interfering with the epigenetic landscape in PDAC has recently been reported for a subgroup of basal-like PDAC subtypes harboring mutations in the gene encoding for the histone demethylase KDM6A. Mechanistically, loss of KDM6A activity results in aberrant activation of superenhancers that regulate the $\triangle N p 63, R U N X$, and MYC oncogenes, thus fostering dedifferentiation and metastasis [20]. Importantly, the increased superenhancer activity renders KDM6A-deficient PDAC more susceptible to inhibition of bromodomain and extra-terminal motif (BET) proteins, which reverses basal-like differentiation and restrains PDAC growth both in vitro and in vivo (Fig. 1) [20]. Using preclinical PDAC models, BET-protein inhibition combined with blockade of histone deacetylase (HDAC) activity has been previously introduced as a promising concept in PDAC treatment [27], albeit without considering the PDAC subtype state. Hence, the stratification for $K D$ -
M6A might even increase the efficacy of BET inhibition for PDAC treatment.

Together, interference with epigenetic regulators to target subtype-specific pathways and expression profiles offers strategies to conquer drug resistance and improve the outcome of PDAC patients. However, the safe and effective application of epigenetic targeting strategies to interfere with PDAC subtype identity requires a more detailed picture reflecting the entire complexity of PDAC molecular heterogeneity. Moreover, regulation of molecular PDAC subtype identity is not restricted to the epithelial tumor cell but is highly influenced by the interaction with other cellular compartments of the tumor.

\section{Targeting Epigenetics to Interfere with Cellular PDAC Heterogeneity}

The cellular heterogeneity of PDAC is based on a pronounced tumor microenvironment (TME) that already forms during pancreatic carcinogenesis and evolves during tumor progression [28]. The PDAC TME, which makes up to $90 \%$ of the tumor bulk, comprises cellular (e.g., fibroblasts and immune cells) and acellular components (e.g., collagen and hyaluronic acid), and intensive biochemical interactions exist between these different compartments and the epithelial tumor cells $[29,30]$. Im- 


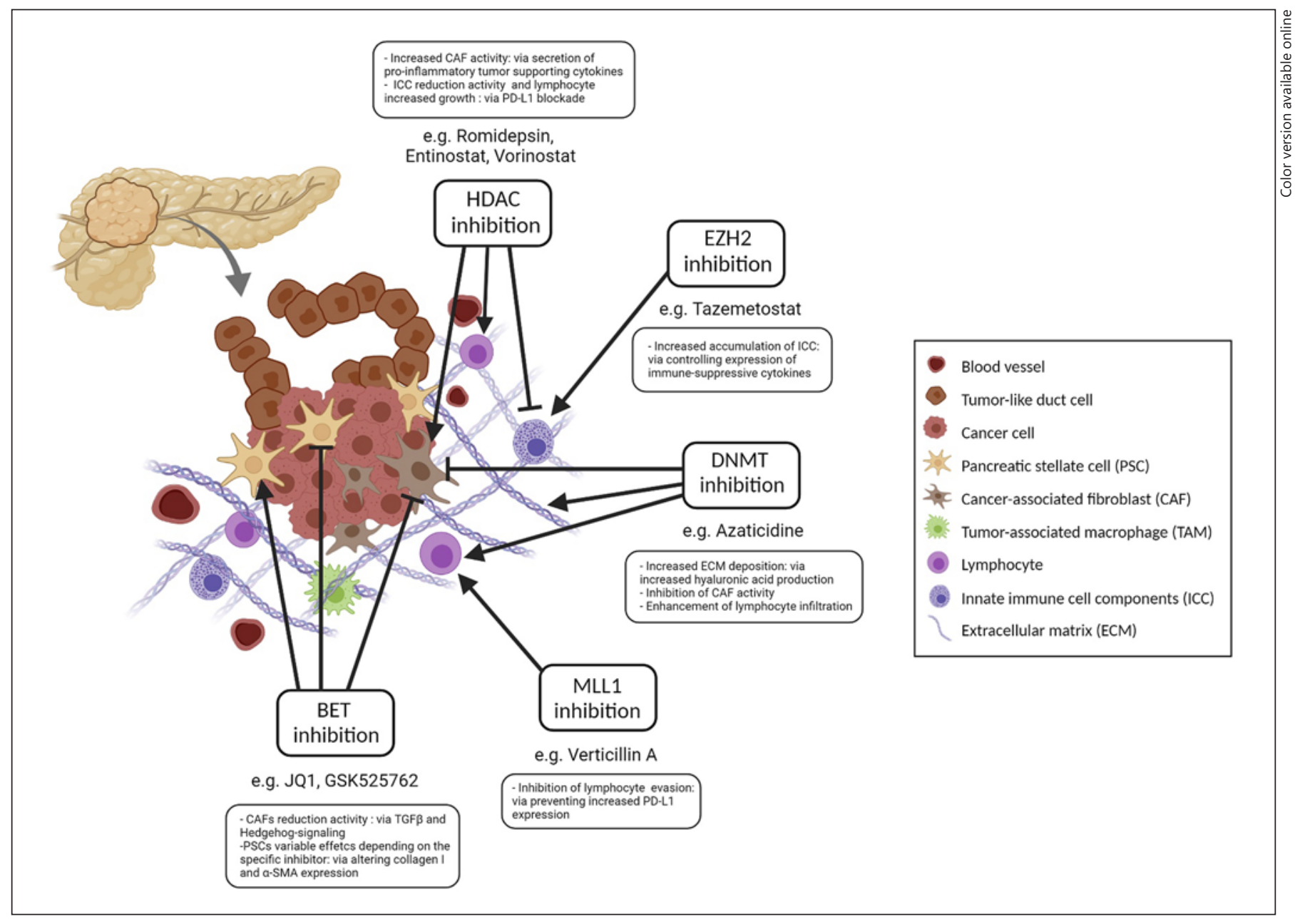

Fig. 2. Epigenetic targeting strategies of the PDAC TME. The main challenge in utilizing epigenetic therapeutic strategies to tackle the cellular heterogeneity of PDAC is associated with the complex and multifactorial role of epigenetic regulation occurring in the different cellular PDAC compartments. Consequently, inhibition of epigenetic regulators can shift the cellular composition of the TME toward a more or less tumor promoting stroma composition. The cartoon was created with BioRender.com. PDAC, pancreatic ductal adenocarcinoma; BET, bromodomain and extra-terminal motif; TME, tumor microenvironment; PSC, pancreatic stellate cell; CAF, cancer-associated fibroblast; TAM, tumor-associated macrophage; ICC, innate immune cell components; ECM, extracellular matrix; HDAC, histone deacetylase.

anisms play a pivotal role in controlling the dynamic plasticity of the cellular components of the PDAC stroma and hence represent promising targets to interfere with the cellular heterogeneity of the disease.

\section{Epigenetic Treatment Strategies to Target the PDAC} TME

Several studies have investigated the role of DNA methylation processes in the regulation of gene expression in multiple components of the TME. For instance, blockade of DNA methylation by applying the DNA methyltransferase (DNMT) inhibitor 5-azacytidine reduced PDAC progression by interfering with global DNA methylation in epithelial PDAC cells and cancer-associated fibroblasts (CAFs) (Fig. 2) [36]. Further supporting the utility of DNMT inhibition in PDAC TME repro- 
gramming, DNMT blockade in immunocompetent PDAC models enhanced $\mathrm{CD} 4^{+}$and $\mathrm{CD} 8^{+} \mathrm{T}$-cell infiltration and caused significant tumor regression (Fig. 2) [37]. However, in contrast to these findings, pharmacological or genetic depletion of DNMT1 resulted in increased production of hyaluronic acid, thus promoting PDAC progression (Fig. 2) [38]. A correlation of low DNA methylation levels and poor PDAC patient outcome has recently also been reported by Espinet et al. [39]. They demonstrate that tumors with a low global DNA methylation in the epithelial cells are characterized by a higher expression of endogenous retroviral transcripts and a strong engagement of the double-stranded RNA sensing machinery with subsequent activation of an interferon signature, thus resulting in pro-tumorigenic reprogramming of stromal cells in the PDAC TME [39]. These contrary findings on the role of DNA methylation in different cellular compartments of the TME suggest that balancing epigenetic treatment strategies is very crucial to find the optimal antitumor composition of epigenetic drugs.

Similar to the implication in driving molecular PDAC subtype identity $[8,15]$, enhancers play a pivotal role in PDAC TME cell specification. Indeed, enhancers have been characterized as "entry gates" for external signaling cues that promote transcription programs required to establish and maintain a TME composition, which fosters tumor development and progression [28, 40, 41]. BET proteins represent critical cofactors that promote (super)enhancer activity and play a pivotal role in PDAC progression, not only in epithelial tumor cells but also in the PDAC stroma $[27,42,43]$. Consequently, BET inhibition decreases TGF $\beta$ and hedgehog signaling, thus reducing the activity of CAFs (Fig. 2), which harbor significant implications in promoting $\mathrm{PDAC}$ progression and therapy resistance $[28,44]$. It is worth noticing that the various BET inhibitors available for clinical or preclinical studies do have distinct specificities for the different BET protein family members BRD2, 3 , and $4[45,46]$. Given that BRD 4 and BRD2/3 promote and block collagen I expression in pancreatic stellate cells, respectively [47] (Fig. 2), a careful selection of these inhibitors based on their target BET protein specificity might be of particular clinical implication. In addition to selecting the right BET inhibitor to combat PDAC, BET inhibition seems to be particularly efficient when combined with the standard chemotherapeutic agent in PDAC treatment, gemcitabine $[27,48]$, or other inhibitors of epigenetic regulators. The combination with HDAC inhibitors, for instance, showed very promising results in preclinical PDAC models [27], although HDAC inhibition in PDAC per se has been linked to the secretion of pro-inflammatory tumor-supporting cytokines by CAFs, thus promoting a tumor-supportive phenotype (Fig. 2) [49]. These findings emphasize the complexity of epigenetic targeting strategies in general, and in remodeling the pancre- atic stroma, in particular. With regard to targeting HDAC proteins, this complexity is even increased given the number of HDAC protein family members and their respective diverse functional involvements [50]. For instance, HDAC proteins are not only mediating HDAC and hence transcriptional regulation but also interfere with posttranslational acetylation of a plethora of target proteins [51]. Consequently, the global and partially unpredictable effects of the diverse HDAC proteins and, therefore of their inhibition, represent a significant obstacle for HDAC inhibition in PDAC treatment. Hence, and in the interest of higher drug specificities, currently active clinical trials exploring HDAC inhibition in PDAC treatment concentrate on HDAC class I and II specific inhibitors (Table 1).

$\mathrm{EZH} 2$ is another chromatin regulatory protein, which directs TME-reprogramming processes in PDAC. Interestingly, and as illustrated in Ezh2-deficient and Krasmutant transgenic mouse models of PDAC, conditional Ezh2 deficiency resulted in increased accumulation of $\mathrm{CD} 11 \mathrm{~b}+$ macrophages, Gr-1+ neutrophils, and CD11c+ dendritic cells in the pancreas, indicating a higher recruitment of innate immune system players (Fig. 2) [52, 53]. These shifts in the immune compartment of the TME were accompanied by increased collagen deposition and aSMA expression and strongly promoted pancreatic carcinogenesis [52]. Given that the ablation of Ezh2 in this model specifically occurred in the epithelial, but not in the stromal cells, these data further imply a strong communication between the epithelial and the TME cellular components of the pancreas. This note is further supported by the fact that the application of the Cox2 inhibitor nimesulide rescued the inflammatory response in these mice and prevented the formation of advanced PDAC precursor lesions [52]. The observations made in the Ezh2-deficient transgenic model are accomplished by several reports, indicating the critical involvement of epigenetic players in controlling immune cell regulation in PDAC development in progression. For instance, epigenetic mechanisms impact on antigen processing and presentation by tumors cells, control the transcription of immune-suppressive cytokines, and impair cytotoxic T-cell function [54]. Accordingly, the CD274 gene, encoding for the immune checkpoint inhibitor PD-L1, is heavily enriched for the H3K4me3 histone mark, rendering its promoter transcriptionally active. This histone mark is installed by the methyltransferase MLL1. Consequently, MLL1 inhibition has been reported to partially prevent increased PD-L1 expression and subsequent immune cell evasion [55]. A similar efficacy in overcoming immune cell evasion in PDAC has been shown upon combining the checkpoint inhibitors anti-PD-1 or anti-CTLA-4 with HDAC inhibition. This combinatory treatment strategy led to a significant abundance of cytotoxic $\mathrm{T}$ cells by decreasing the activity of 
myeloid-derived suppressor cells in the TME (Fig. 2) [56]. This report strongly argues for therapeutic strategies in which epigenetic treatment concepts are utilized to prime the immune PDAC TME for the subsequent exposure to immunomodulatory drugs. A sequential approach based on HDAC/DNMT inhibition combined with chemotherapy and followed by PD-L1 blockade is currently undergoing clinical testing in PDAC (NCT04257448, Table 1), and results are eagerly awaited.

\section{Conclusion}

Without doubt, epigenetic regulatory proteins represent pivotal drivers of PDAC tumor progression and therapy resistance, not only, but particularly by determining the cellular and molecular heterogeneity of the disease. Translational studies conducted within the last years have convincingly demonstrated the utility of epigenetic targeting in interfering with PDAC plasticity and switching between different cellular and molecular states. Nevertheless, in contrast to other tumor entities, epigenetic targeting of PDAC is still in its infancy. This is also indicated by the relatively low number of epigenetic drugs which qualify for clinical testing in PDAC (Table 1). Despite significant efforts, important technical advances and the increasing understanding of the implications of PDAC heterogeneity for the disease course and outcome, we have only started to dissect the molecular underpinnings and the upstream regulatory components, which finally give rise to the complex epigenetic landscape evident in PDAC. The dynamic nature of epigenetic alterations allows reverting cellular states and conditions in favor of a less aggressive and more therapy susceptible molecular and cellular makeup of PDAC. However, epigenetic mechanisms often play contrary roles in different cellular components of PDAC, with tumor-promoting activities in one, and tumor-suppressive functions in other compartments. Unfortunately, current epigenetic targeting strategies cannot distinguish between the different functional implications of spatially distinct but otherwise identical, epigenetic mechanisms.

In contrast to hematological malignancies in which epigenetic treatment strategies play an increasing role even in monotherapeutic application, it does not seem likely that targeting of 1 epigenetic regulator or even of a family of epigenetic proteins is sufficient to combat PDAC. As described in this article, first promising results have been reported for combinatory treatment strategies, which involve epigenetic targeting. From these studies, we gained first evidence that epigenetic therapies have a strong potential in priming PDAC tumor cells and their TME for additional, predominantly cytotoxic, therapy. Hence, in addition to further disentangle the complex interactions between epigenetic mechanisms and additional regulatory processes evident in PDAC, translational efforts need to unravel the best sequence of epigenetic drug application to combat this dismal disease.

\section{Conflict of Interest Statement}

The authors have no conflict of interest to declare.

\section{Funding Sources}

This work has been supported by the German Research Foundation (DFG, to E.H.: KFO5002) and the German Cancer Aid (to E.H.: 70114087).

\section{Author Contributions}

L.V. wrote the part on epigenetics in PDAC molecular heterogeneity and drafted Figure 1. M.U. wrote the part on PDAC cellular heterogeneity and designed Figure 2 and Table 1. E.H. developed the review's concept and structure, wrote the introduction and the conclusion, and compiled all sections of this review.

\section{References}

1 Waddell N, Pajic M, Patch AM, Chang DK, Kassahn KS, Bailey P, et al. Whole genomes redefine the mutational landscape of pancreatic cancer. Nature. 2015;518(7540):495-501.

2 Chan-Seng-Yue M, Kim JC, Wilson GW, Ng K, Figueroa EF, O’Kane GM, et al. Transcription phenotypes of pancreatic cancer are driven by genomic events during tumor evolution. Nat Genet. 2020;52(2):231-40.

3 Bailey P, Chang DK, Nones K, Johns AL, Patch AM, Gingras MC, et al. Genomic analyses identify molecular subtypes of pancreatic cancer. Nature. 2016;531(7592):47-52.

4 Collisson EA, Sadanandam A, Olson P, Gibb WJ, Truitt M, Gu S, et al. Subtypes of pancreatic ductal adenocarcinoma and their differing responses to therapy. Nat Med. 2011;17(4):500-3.
5 Moffitt RA, Marayati R, Flate EL, Volmar KE, Loeza SG, Hoadley KA, et al. Virtual microdissection identifies distinct tumor- and stromaspecific subtypes of pancreatic ductal adenocarcinoma. Nat Genet. 2015;47(10):1168-78.

6 Raphael BJ, Hruban RH, Aguirre AJ, Moffitt RA, Yeh JJ, Stewart C, et al. Integrated genomic characterization of pancreatic ductal adenocarcinoma. Cancer Cell. 2017;32(2):185-203.e13.

7 Puleo F, Nicolle R, Blum Y, Cros J, Marisa L, Demetter P, et al. Stratification of pancreatic ductal adenocarcinomas based on tumor and microenvironment features. Gastroenterology. 2018;155(6):1999-2013.e3.

8 Lomberk G, Blum Y, Nicolle R, Nair A, Gaonkar KS, Marisa L, et al. Distinct epigenetic landscapes underlie the pathobiology of pan- creatic cancer subtypes. Nat Commun. 2018; 9(1):1978.

9 Hessmann E, Johnsen SA, Siveke JT, Ellenrieder V. Epigenetic treatment of pancreatic cancer: is there a therapeutic perspective on the horizon? Gut. 2017;66(1):168-79.

10 Alexandrov LB, Nik-Zainal S, Siu HC, Leung SY, Stratton MR. A mutational signature in gastric cancer suggests therapeutic strategies. Nat Commun. 2015;6:8683.

11 Bettaieb A, Paul C, Plenchette S, Shan J, Chouchane L, Ghiringhelli F. Precision medicine in breast cancer: reality or utopia? J Transl Med. 2017;15(1):139.

12 Politi K, Herbst RS. Lung cancer in the era of precision medicine. Clin Cancer Res. 2015; 21(10):2213-20. 
13 Biankin AV, Waddell N, Kassahn KS, Gingras MC, Muthuswamy LB, Johns AL, et al. Pancreatic cancer genomes reveal aberrations in axon guidance pathway genes. Nature. 2012; 491(7424):399-405.

14 Aung KL, Fischer SE, Denroche RE, Jang GH, Dodd A, Creighton S, et al. Genomics-driven precision medicine for advanced pancreatic cancer: early results from the COMPASS Trial. Clin Cancer Res. 2018;24(6):1344-54.

15 Lomberk G, Dusetti N, Iovanna J, Urrutia R. Emerging epigenomic landscapes of pancreatic cancer in the era of precision medicine. Nat Commun. 2019;10(1):3875.

16 Pott S, Lieb JD. What are super-enhancers? Nat Genet. 2015;47(1):8-12.

17 Whyte WA, Orlando DA, Hnisz D, Abraham BJ, Lin CY, Kagey MH, et al. Master transcription factors and mediator establish super-enhancers at key cell identity genes. Cell. 2013; 153(2):307-19.

18 Hamdan FH, Johnsen SA. DeltaNp63-dependent super enhancers define molecular identity in pancreatic cancer by an interconnected transcription factor network. Proc Natl Acad Sci U S A. 2018;115(52):E12343-52.

19 Somerville TDD, Xu Y, Miyabayashi K, Tiriac H, Cleary CR, Maia-Silva D, et al. TP63-mediated enhancer reprogramming drives the squamous subtype of pancreatic ductal adenocarcinoma. Cell Rep. 2018;25(7):1741-55.e7.

20 Andricovich J, Perkail S, Kai Y, Casasanta N, Peng W, Tzatsos A. Loss of KDM6A activates super-enhancers to induce gender-specific squamous-like pancreatic cancer and confers sensitivity to BET inhibitors. Cancer Cell. 2018;33(3):512-26.e8.

21 Patil S, Steuber B, Kopp W, Kari V, Urbach L, Wang X, et al. EZH2 regulates pancreatic cancer subtype identity and tumor progression via transcriptional repression of GATA6. Cancer Res. 2020;80(21):4620-32.

22 Kloesch B, Ionasz V, Paliwal S, Hruschka N, Martinez de Villarreal J, Öllinger R, et al. A GATA6-centred gene regulatory network involving HNFs and $\triangle \mathrm{Np} 63$ controls plasticity and immune escape in pancreatic cancer. Gut. 2021.

23 Hui CC, Angers S. Gli proteins in development and disease. Annu Rev Cell Dev Biol. 2011;27:513-37.

24 Adams CR, Htwe HH, Marsh T, Wang AL, Montoya ML, Subbaraj L, et al. Transcriptional control of subtype switching ensures adaptation and growth of pancreatic cancer. eLife. 2019;8:e45313.

25 Daemen A, Peterson D, Sahu N, McCord R, Du X, Liu B, et al. Metabolite profiling stratifies pancreatic ductal adenocarcinomas into subtypes with distinct sensitivities to metabolic inhibitors. Proc Natl Acad Sci U S A. 2015;112(32):E4410-7.

26 Brunton H, Caligiuri G, Cunningham R, Upstill-Goddard R, Bailey UM, Garner IM, et al. HNF4A and GATA6 loss reveals therapeutically actionable subtypes in pancreatic cancer. Cell Rep. 2020;31(6):107625.

27 Mazur PK, Herner A, Mello SS, Wirth M, Hausmann S, Sánchez-Rivera FJ, et al. Combined inhibition of BET family proteins and histone deacetylases as a potential epigenetics-based therapy for pancreatic ductal adenocarcinoma. Nat Med. 2015;21(10): 1163-71.

28 Hessmann E, Buchholz SM, Demir IE, Singh SK, Gress TM, Ellenrieder V, et al. Microenvironmental determinants of pancreatic cancer. Physiol Rev. 2020;100(4):1707-51.

29 Neesse A, Algül H, Tuveson DA, Gress TM. Stromal biology and therapy in pancreatic cancer: a changing paradigm. Gut. 2015; 64(9):1476-84.

30 Huber M, Brehm CU, Gress TM, Buchholz M, Alashkar Alhamwe B, von Strandmann EP, et al. The immune microenvironment in pancreatic cancer. Int J Mol Sci. 2020;21(19): 7307.

31 Jacobetz MA, Chan DS, Neesse A, Bapiro TE, Cook N, Frese KK, et al. Hyaluronan impairs vascular function and drug delivery in a mouse model of pancreatic cancer. Gut. 2013; 62(1):112-20.

32 Hessmann E, Patzak MS, Klein L, Chen N, Kari V, Ramu I, et al. Fibroblast drug scavenging increases intratumoural gemcitabine accumulation in murine pancreas cancer. Gut. 2018;67(3):497-507.

33 Buchholz SM, Goetze RG, Singh SK, AmmerHerrmenau C, Richards FM, Jodrell DI, et al. Depletion of macrophages improves therapeutic response to gemcitabine in murine pancreas cancer. Cancers. 2020;12(7):1978.

34 Neesse A, Bauer CA, Öhlund D, Lauth M, Buchholz M, Michl P, et al. Stromal biology and therapy in pancreatic cancer: ready for clinical translation? Gut. 2019;68(1):159-71.

35 Guo S, Contratto M, Miller G, Leichman L, $\mathrm{Wu}$ J. Immunotherapy in pancreatic cancer: unleash its potential through novel combinations. World J Clin Oncol. 2017;8(3):230-40.

36 Shakya R, Gonda T, Quante M, Salas M, Kim $\mathrm{S}$, Brooks J, et al. Hypomethylating therapy in an aggressive stroma-rich model of pancreatic carcinoma. Cancer Res. 2013;73(2):885-96.

37 Ebelt ND, Zuniga E, Johnson BL, Diamond DJ, Manuel ER. 5-Azacytidine potentiates anti-tumor immunity in a model of pancreatic ductal adenocarcinoma. Front Immunol. 2020;11:538.

38 Kohi S, Sato N, Cheng XB, Koga A, Higure A, Hirata K. A novel epigenetic mechanism regulating hyaluronan production in pancreatic cancer cells. Clin Exp Metastasis. 2016;33(3): 225-30.

39 Espinet E, Gu Z, Imbusch CD, Giese NA, Büscher M, Safavi M, et al. Aggressive PDACs show hypomethylation of repetitive elements and the execution of an intrinsic ifn program linked to a ductal cell of origin. Cancer Discov. 2021;11(3):638-59.

40 Gosselin D, Link VM, Romanoski CE, Fonseca GJ, Eichenfield DZ, Spann NJ, et al. Environment drives selection and function of enhancers controlling tissue-specific macrophage identities. Cell. 2014;159(6):1327-40.

41 Lavin Y, Winter D, Blecher-Gonen R, David E, Keren-Shaul H, Merad M, et al. Tissue-resident macrophage enhancer landscapes are shaped by the local microenvironment. Cell. 2014;159(6):1312-26.

42 Garcia PL, Miller AL, Kreitzburg KM, Council LN, Gamblin TL, Christein JD, et al. The BET bromodomain inhibitor JQ1 suppresses growth of pancreatic ductal adenocarcinoma in patient-derived xenograft models. Oncogene. 2016;35(7):833-45

43 Sahai V, Kumar K, Knab LM, Chow CR, Raza SS, Bentrem DJ, et al. BET bromodomain inhibitors block growth of pancreatic cancer cells in three-dimensional collagen. Mol Cancer Ther. 2014;13(7):1907-17.

44 Yamamoto K, Tateishi K, Kudo Y, Hoshikawa M, Tanaka M, Nakatsuka T, et al. Stromal remodeling by the BET bromodomain inhibitor JQ1 suppresses the progression of human pancreatic cancer. Oncotarget. 2016;7(38): 61469-84.

45 Filippakopoulos P, Knapp S. Targeting bromodomains: epigenetic readers of lysine acetylation. Nat Rev Drug Discov. 2014;13(5):337-56.

46 Filippakopoulos P, Qi J, Picaud S, Shen Y, Smith WB, Fedorov O, et al. Selective inhibition of BET bromodomains. Nature. 2010; 468(7327):1067-73.

47 Kumar K, DeCant BT, Grippo PJ, Hwang RF, Bentrem DJ, Ebine K, et al. BET inhibitors block pancreatic stellate cell collagen I production and attenuate fibrosis in vivo. JCI Insight. 2017;2(3):e88032.

48 Baretti M, Ahuja N, Azad NS. Targeting the epigenome of pancreatic cancer for therapy: challenges and opportunities. Ann Pancreatic Cancer. 2019;2.

49 Nguyen AH, Elliott IA, Wu N, Matsumura C, Vogelauer M, Attar N, et al. Histone deacetylase inhibitors provoke a tumor supportive phenotype in pancreatic cancer associated fibroblasts. Oncotarget. 2017;8(12):19074-88.

50 Schneider G, Krämer OH, Fritsche P, Schüler S, Schmid RM, Saur D. Targeting histone deacetylases in pancreatic ductal adenocarcinoma. J Cell Mol Med. 2010;14(6a):1255-63.

51 Kulka LAM, Fangmann PV, Panfilova D, Olzscha H. Impact of HDAC inhibitors on protein quality control systems: consequences for precision medicine in malignant disease. Front Cell Dev Biol. 2020;8(425):425.

52 Mallen-St Clair J, Soydaner-Azeloglu R, Lee KE, Taylor L, Livanos A, Pylayeva-Gupta Y, et al. EZH2 couples pancreatic regeneration to neoplastic progression. Genes Dev. 2012; 26(5):439-44

53 Chen NM, Neesse A, Dyck ML, Steuber B, Koenig AO, Lubeseder-Martellato C, et al. Contextdependent epigenetic regulation of nuclear factor of activated T cells 1 in pancreatic plasticity. Gastroenterology. 2017;152(6):1507-20.e15.

54 Héninger E, Krueger TE, Lang JM. Augmenting antitumor immune responses with epigenetic modifying agents. Front Immunol. 2015;6:29.

55 Lu C, Paschall AV, Shi H, Savage N, Waller JL Sabbatini ME, et al. The MLL1-H3K4me3 axis-mediated PD-L1 expression and pancreatic cancer immune evasion. J Natl Cancer Inst. 2017;109(6):djw283.

56 Christmas BJ, Rafie CI, Hopkins AC, Scott BA, Ma HS, Cruz KA, et al. Entinostat converts immune-resistant breast and pancreatic cancers into checkpoint-responsive tumors by reprogramming tumor-infiltrating MDSCs. Cancer Immunol Res. 2018;6(12):1561-77.

57 Gerrard DL, Boyd JR, Stein GS, Jin VX, Frietze S. Disruption of broad epigenetic domains in PDAC cells by HAT inhibitors. Epigenomes. 2019;3(2). 